\title{
Empleo del estropajo común (Luffa cylindrica) en la remoción de contaminantes
}

\section{Use of the common sponge (Luffa cylindrica) in the removal of contaminants}

\author{
Usando a bucha comum (Luffa cylindrica) \\ na remoção de contaminantes
}

\author{
Ricardo Ignacio Pereira Martínez', Juan Fernando Muñoz Paredes ${ }^{2}$ \\ \& Diego Hernán Peluffo Ordoñez ${ }^{3}$. \\ 'Ingeniero industrial, Estudiante de maestría en Ingeniería Ambiental. ${ }^{2}$ Ingeniero Químico, MSc en Ingeniería \\ Ambiental. ${ }^{3}$ Ingeniero Electrónico, magister en Ingeniería y PhD en Automatización Industrial. \\ ${ }^{1}$ Grupo de investigación ESLINGA de Ingeniería Industrial. Universidad Cooperativa de Colombia. Pasto. Pasto \\ Colombia. ${ }^{2}$ Facultad de Posgrados y Relaciones Internacionales Universidad Mariana. Pasto. Colombia. 2Grupo de \\ investigación GIA - Grupo de Investigación Cambio climático y sostenibilidad. Universidad Mariana. Pasto. \\ Colombia. ${ }^{3} U$ niversidad Técnica del Norte. Ibarra. Ecuador. \\ 1ricardo.pereira@ucc.edu.co, 2juanfemp@hotmail.com, ${ }^{3}$ diegohpo@gmail.com
}

\section{Resumen}

Interesa a la ingeniería, el desarrollo biotecnológico de técnicas de tratamiento de agua para la remoción de contaminantes, aprovechando las propiedades de las fibras de Luffa cylindrica (FLc). Así lo exponen los argumentos explicados en varias investigaciones realizadas en todo el mundo en las que se describe un sugestivo escenario de razones válidas para considerar a la fibra del estropajo, como un material industrialmente promisorio y sostenible, apto para la realización de tratamientos de remoción de contaminantes y en la separación de sustancias inmersas en matrices fluidas. También explican la utilización de las fibras como matriz inmovilizadora para sostener comunidades microbianas activas implantadas con fines específicos; incluso, al comprender la arquitectura y las propiedades mecánicas de las FLc, se explora su utilización como agregado en la obtención de materiales compuestos, en la producción de nuevas sustancias y su capacidad para retener humedad. De modo que el enfoque en el que se ha interesado el presente trabajo se refiere a la descripción de los procesos de adsorción e inmovilización en los cuales se ha involucrado a las FLc haciendo una revisión de experiencias investigativas. A la vez se estudian, los razonamientos que han permitido describir las técnicas y que posibilitan el aporte de soluciones al problema de la remoción de contaminantes y del tratamiento de agua.

Palabras clave: biofiltro, estropajo, adsorbente, esponja vegetal, fibras, nanofibras.

\section{Abstract}

It is in the interest of the engineering, the biotechnological development of techniques for the treatment of water for the removal of contaminants, taking 
advantage of the properties of the fibers of Luffa cylindrica (FLC). So exposed the arguments explained in several investigations carried out throughout the world in which describes a suggestive scene of valid reasons to consider the fiber of the sponge, as a material industrially promising and sustainable, suitable for the realization of removal treatments of contaminants and on the separation of substances immersed in arrays fluid. They also explain the use of fibers such as array immobilising to sustain microbial communities active implanted with specific purposes; even to understand the architecture and the mechanical properties of the FLc, explores its use as an aggregate in the obtaining of composite materials in the production of new substances and their capacity to retain moisture. Therefore, the approach that has been interested in the present work refers to the description of the description of the processes of adsorption and immobilization in which has been involved in the FLc doing a review of experiences research. At the same time, the reasons that have allowed describe techniques and that makes it possible to provide solutions to the problem of contaminant removal and treatment of water, are studied.

Key-words: biofilter, scourer, adsorbent, sponge vegetable fibers, nanofibers

\section{Resumo}

É de interesse da engenharia o desenvolvimento biotecnológico de técnicas de tratamento de águas para remover contaminantes, aproveitando as propriedades das fibras de Luffa cylindrica (FLc). É assim que têm sido mostrado em numerosas pesquisas ao redor do mundo, onde é descrito um sugestivo cenário de vários argumentos para considerar a fibra da bucha como material industrialmente promissório e sustentável para a realização de tratamentos para remover contaminantes e separar substancias submersas em matrizes fluidas. Também explicam o uso das fibras como imobilizadores de comunidades microbianas ativas com uma finalidade específica. Ao compreender a arquitetura e as propriedades mecânicas das FLc, explora-se seu uso como agregado na obtenção de materiais compostos, na produção de substâncias novas e sua capacidade para reter umidade. Nesse contexto, o foco do deste trabalho é a descrição dos processos de adsorção e imobilização onde involucram-se as FLc, fazendo uma revisão das experiências de pesquisas. Ao mesmo tempo, é feito o estudo das bases que têm permitido descrever as técnicas e que possibilitam soluções ao problema de remover contaminantes e do tratamento da água.

Palavras-chave: biofiltro, bucha, adsorvente, esponja vegetal, fibras, nanofibras.

\section{Introducción}

Además de la revisión de los conocimientos alcanzados por las investigaciones dedicadas a evaluar la aplicabilidad de las fibras de Luffa cylindrica FLc en diferentes tratamientos de remoción de igualmente diversos tipos de contaminantes, este artículo, al efectuar un estudio metódico de las publicaciones científicas del ámbito internacional, ha pretendido hacer un recuento de investigaciones dedicadas al estudio de las FLc en la remoción de contaminantes y recopilar sus resultados, para exponer, con un estilo descriptivo, las aplicaciones metodológicas de los tratamientos, las técnicas analíticas y los resultados alcanzados; de manera que la finalidad última de esta investigación de revisión sea la difusión del conocimiento referente a la eficiencia de los procesos y a las conclusiones experimentales en la cinemática de remoción de contaminantes empleando a las FLc como agente removedor o portante según sea cada caso.

También se han indagado experiencias dedicadas a conocer la estructura y propiedades físicas de las fibras, procurando con ello, comprender las virtudes y características del material a la vez que se escruta una posible relación entre la estructura y los fenómenos ocurridos durante los tratamientos de remoción estudiados, de manera que se puedan inferir nuevas hipótesis y preguntas en torno al estudio del material fibroso. Finalmente se ha encontrado que a la luz de 
las experiencias científicas referidas, los argumentos expuestos, respaldan la utilidad potencial que tienen estas nano-fibras en los procesos de remoción de contaminantes complejos de manera sostenible y sustentable; se estima a las FLc como un material con alta utilidad práctica para la industria y la sociedad; necesidades que justifican el quehacer investigativo sobre la capacidad de la luffa para remover más sustancias del inventario de contaminantes y aplicar sus resultados al diseño de instrumentos de separación y tratamiento, con aplicación industrial, doméstica y social.

\section{Metodología}

Para la adecuada revisión del tema relacionado con los tratamientos de remoción de contaminantes, utilizando las fibras de Luffa cylindrica, se han tamizado 48 artículos científicos que fueron publicados por investigadores adscritos a universidades, institutos o centros de investigación de todo el mundo, en idioma inglés, español y portugués, disponibles en revistas científicas de reconocido prestigio y confiabilidad internacional con disponibilidad virtual, seleccionando los artículos producidos con posterioridad al año 2000, aunque vale aclarar la excepción hecha con el trabajo publicado por Liu, Seki, Tanaka \& Furusaky en 1998. También se estudiaron algunos artículos relativos a la botánica de la luffa, la estructura del estropajo y las propiedades mecánicas de las FLc.

\section{Rasgos botánicos, estructurales y mecánicos de las FLc.}

Comúnmente llamada "estropajo", esta planta es miembro de la familia de las cucurbitáceas en las que se reconocen siete especies del género Luffa (Shapiro, 2012). Sus hojas alternas y palmeadas son lizas con bordes dentados y la flor es de color amarillo. La planta es que es monoica (UNAD, 2013) y sus frutos son una calabaza lisa, oblonga en forma de cilindro que tiene entre 40 a $70 \mathrm{~cm}$ de largo que exhibe líneas verticales en su exterior. En su mesocarpio se forma una intrincada retícula fibrosa constituida por lignocelulosa que es la parte típicamente útil de la planta (Luffa.info, 2014). El estropajo es una importante planta que provee de alternativas de aplicación diversas en salud (Azeez, Bello, \& Adedeji, 2013) y que plantea promisorias utilizaciones en la tecnología de los materiales (Shen, Xie, Huang, Zohu, \& Ruan, 2013).

Investigaciones dedicadas al estudio de la morfología del estropajo (Figura 1) reportaron datos dimensionales similares de las fibras que inducen a pensar que podría existir una importante relación entre la forma y la estructura de la fibra con sus propiedades adsorbentes y mecánicas; supuesto que resulta muy pertinente con la tecnología que pretende el aprovechamiento de la esponja, de allí la importancia de su descripción.

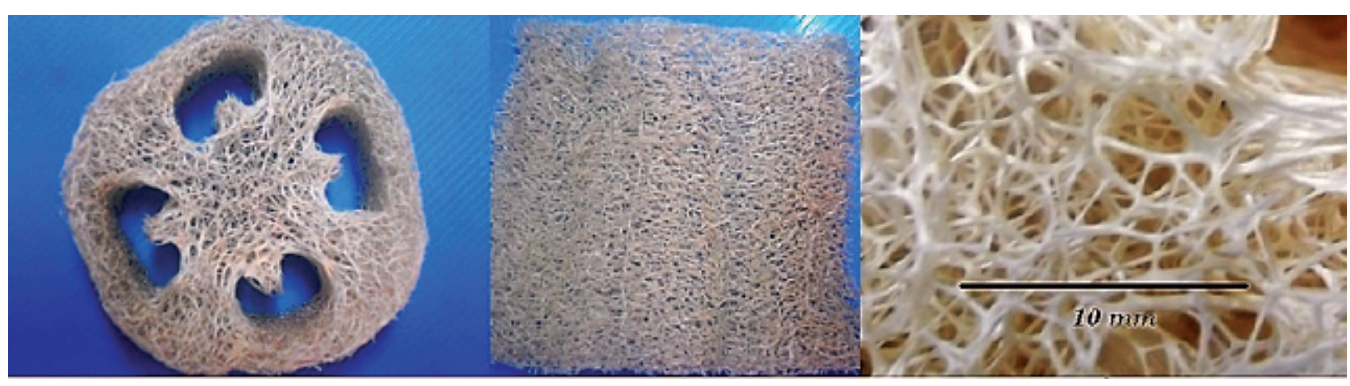

Figura 1. Morfología estructural de las FLc crudas.

Se describe que la esponja de FLc, superpone una serie de tres capas fibrosas, flexibles y fuertes que se unen en mediante puntales esponjosos longitudinales a un tallo central formando por una estructura ramificada de fibras más gruesas (Cifuentes \& Pataquiva, 2013). Las fibras de la capa externa tienen una longitud media de $1 \mathrm{~mm}$ que se extienden densamente transversales al eje del fruto; mientras que 
las fibras del tallo central -capa interior-, son más largas próximas a los $5 \mathrm{~mm}$ que se extienden desde su centro hacia la capa exterior pasando por una capa intermedia de unión (Shen, Yi, Huang, Zhou, \& Ruan, 2012). Las fibras, se aprecian formadas por un entramado de micro-tubos coriáceos caracterizados por una estructura celular de micro canales continuos huecos con diámetros que varían entre 10 y $20 \mu \mathrm{m}$, formando haces vasculares que producen un sistema poroso complejo y extenso (Figura 2) (Altinisk, Gür, \& Seki, 2010). La superficie tubular exhibe finos filamentos que se extienden longitudinalmente sobre el eje de la fibra (Figura 3) (Kaewta \& Thongmee, 2012).
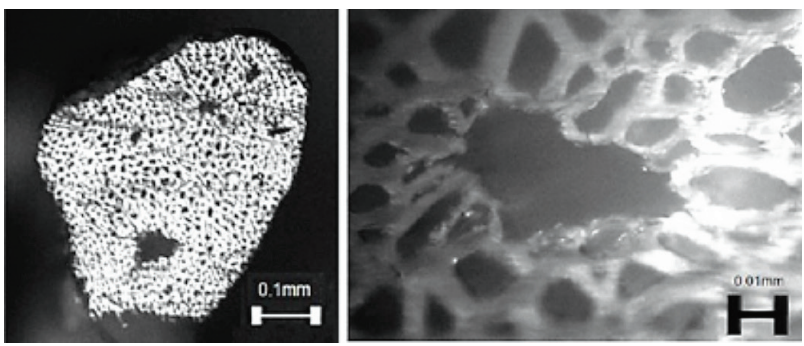

Figura 2. Corte transversal de una fibra de Luffa a $0,1 \mathrm{~mm}$ y vista de estructura nanotubular a $0,01 \mathrm{~mm}$ Fuente: Shen et. al., (2012).

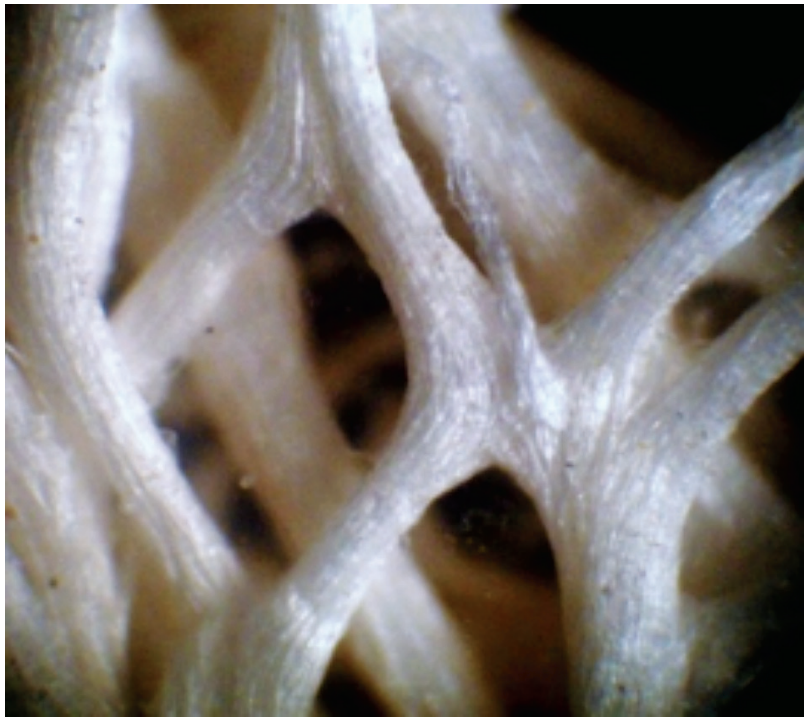

Figura 3. Apariencia superficial de las FLc.

Sostienen además Cifuentes \& Pataquiva que debido a la estructura fibrosa y porosa de las fibras, las esponjas son propensas a adquirir fácilmente impurezas del ambiente. Además, su superficie rugosa y con escamas, facilita la retención de iones metálicos (Lindino, Marciniak, Gonzales Jr., \& Strey, 2014). A estas características se le atribuyen las propiedades adsorbentes que facilitan su uso en las pruebas de remoción y de inmovilización microbiana (Figura 4) (Akhtar, Iqbal, \& Iqbal, 2004).
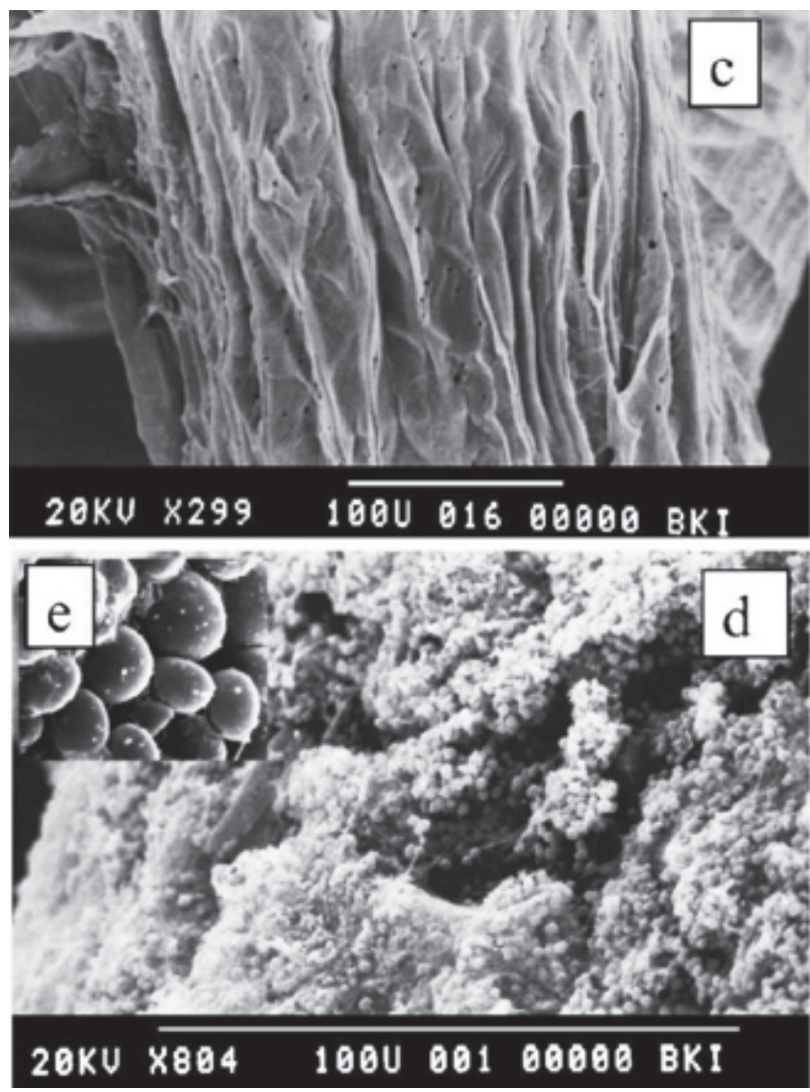

Figura 4. Imagen microscópica de la superficie de las fibras que han inmovilizado partículas metálicas de níquel II (arriba). Algas del género Chlorella sorokiniana inmovilizadas adheridas a la fibra Fuente: Akhtar, Iqbal, \& Iqbal, (2004).

También se reporta que el tratamiento de $\mathrm{NaOH}$ al $2 \%$ mejora la rugosidad superficial de las fibras (Tanobe, Sydenstryker, Munaro, \& Amico, 2004) Igualmente el Instituto Indio de Tecnología de Roorkee, informó sobre el desarrollo de un tratamiento a base de microondas para modificar la textura de las FLc para su posterior aplicación en la adsorción de sustancias (Kumar, Pathania, \& Sharma, 2014).

Estudios relacionados con las propiedades mecánicas de las FLC, han determinado que la densidad de las FLc depende de la capa esponjosa a la 
pertenecen, oscilando su valor entre 350 y $650 \mathrm{~kg} /$ $\mathrm{m}^{3}$, (Shen, Yi, Huang, Zhou, \& Ruan, 2012). Concluyen los mismos investigadores, que las propiedades mecánicas de las FLc son influenciadas principalmente por el grado de humedad presente en el interior de la fibra y agregan que también lo están por su aspecto superficial (Kaewta \& Thongmee, 2012), el cual puede modificarse exponiendo la fibra a soluciones de $\mathrm{NaOH}$, que al desprender su capa externa deja expuesta la superficie rugosa de la fibra lo cual maximiza su interacción superficial con las sustancias en tratamiento y haciendo que la fibra actúe como un material de celda abierta de espuma adsorbente (Boynard, Monteiro, \& d'Almeida, 2002).

\section{Experiencias en el estudio de las propiedades adsorbentes de las FLc.}

En diferentes condiciones de tratamiento, se han puesto a prueba las FLc como elemento adsorbente para remover sustancias de prueba en diferentes matrices fluidas, generalmente agua, con la finalidad de evaluar su eficiencia de remoción y los fenómenos asociados al proceso. A la propiedad adsorbente de las FLc, se le atribuye la capacidad de remoción por intercambio protónico de metales pesados, por cuanto las fibras interactúan electrostáticamente con el oxígeno libre producto de la protonación de las moléculas de las sustancias en proceso, los electrones libres de los aniones metálicos presentes y los iones libres de las mismas sustancias; constituyéndose este fenómeno en el principal mecanismo de adsorción (López, Vasquez, Suárez, \& Gómez , 2012)

La bio-adsorción es un proceso de separación de equilibrio bien conocido y un método eficaz para aplicaciones de descontaminación de agua (Oboh \& Alyour, 2009). En este sentido la Luffa cylindrica es un material ligno-celulósico que se puede utilizar como bio-adsorbente debido a la acción de su sistema vascular fibroso que permite la eliminación de contaminantes del agua, incluidos tintes, metales y sales disueltas (Ye, Hu, \& Wang, 2013), y por la protonación de sus fibras que permite la retención de radicales químicos. La capacidad de inmovilización de microorganismos interactuando con las fibras en la remoción de sustancias orgánicas y minerales no metálicas está ampliamente referenciada, siendo este un tema que se tratará más adelante por cuanto el fenómeno de remoción está asociada a la acción de los microorganismos implantados como residentes en las fibras más que a las propiedades individuales de la luffa. Estas características hacen que se puedan considerar a las FLc como un bio-material promisorio para la producción de elementos de utilidad industrial tales como filtros y membranas, según lo sostienen López, Vasquez, Suárez, \& Gómez (2012). De éste modo los resultados de las investigaciones revisadas concluyen en general, que las fibras de la Luffa cylindrica tienen alta y probada capacidad para retener sustancias contaminantes.

Reportan Adie, Igboro \& Daouda (2013), desde el Departamento de Ingeniería Civil del Pilotécnico de Kaduna en Nigeria, que se experimentó un filtro piloto a base de Luffa cylindrica para observar la eficacia filtrante de la esponja, en un proceso constituido por cuatro etapas arregladas en serie y con diferencial de densidad en dirección del flujo del agua de prueba; concluyendo el reporte con la evidencia de una alta eficiencia hidráulica de la esponja en un filtro horizontal, con una capacidad del $80 \%$ en la inmovilización de microorganismos, una moderada capacidad del $48,33 \%$ en la eliminación de TSS resultando en una remoción de turbidez del $18,15 \%$, del $10 \%$ de la dureza, el $16 \%$ en cloruros, $16,66 \%$ para el nitrógeno amoniacal y ninguna afectación a la alcalinidad moderadamente ácida. Reporta también el mismo estudio, una alta eficiencia en la retención de metales pesados, atribuyendo los anteriores resultados a la porosidad y morfología de las fibras.

Por su parte la remoción de iones metálicos está más asociada a las propiedades adsorbentes de las fibras. En la experiencia realizada por el departamento de ingeniería química de la Universidad de Benin en Nigeria, en la etapa pos-tratamiento de aguas residuales contaminadas con hidrocarburos, se experimentó con una capa de fibra desmenuzada de Luffa cylindrica, en la que se pudo determinar que los porcentajes de eliminación de los iones metálicos presentes en el agua de la muestra se 
estimaron en el $92,31 \%, 88,64 \%, 85,71 \%$ y $66,67 \%$ para Cr3 +, Zn2 +, Pb2 + y Cd2 +, respectivamente (Aluyor, Oboh, \& Adhu, 2009). Vale destacar que los iones relacionados corresponden a metales pesados altamente tóxicos y difíciles de disponer. El mismo estudio pudo establecer que también hubo reducción de los iones de sulfato en un $1,32 \%$, de THC (tetrahidrocanabitol) en un $7,31 \%$, en la salinidad en un $4,79 \%$ y por tanto la conductividad del agua en un $4,08 \%$. Datos que resultan similares con los reportados por Adie, Igboro \& Daouda en 2013.

En otro experimento de remoción con FLc, se utilizó sulfato de cobre y azul de metileno, se analizó la capacidad de adsorción del cobre y la capacidad de regeneración de la fibra, con satisfactorios resultados en ambientes ligeramente ácidos cercanos a pH 5 (Laidan, Hanini, \& Henini, 2010). En esta experiencia, llama la atención el seguimiento de la metodología utilizada por Altınısık, Gür, y Seki, en cuanto a que se aplicaron los modelos de Langmuir y Freundlich que produjeron isotermas que explican satisfactoriamente la eficiencia de remoción del metal, la cinemática y la termodinámica del proceso, resultados que también fueron logradas en la remoción del verde de malaquita (Altinisk, Gür, \& Seki, 2010) y de azul de metileno (Demir, Top, Balköse, \& Ülkü, 2005). Se destaca que en ambos experimentos, la esponja es tratada con $\mathrm{NaOH}$ para su preparación y posterior desmechado en fibras de 1 a $2 \mathrm{~mm}$; proceso que también explicaron Boynard, Monteiro, \& d'Almeida, al referirse a las interacciones superficiales de las fibras puras y sus propiedades en la obtención de materiales compuestos, que a su vez facilitan la retención de iones metálicos por adsorción electrostática con la rugosa superficie fibrosa en la remoción de iones de cadmio (II) (Shajidi, Jalilnejad, \& Jalilnejad, 2013) y en otras descripciones alternativas a la remoción del mismo metal (Purkayastha, Mishra, \& Biswas, 2014). La Luffa cylindrica es un material natural que posee una tasa de retención de los iones de Cadmio en su superficie del 99,80\% a un $\mathrm{pH} 5.0$ en procesos de percolado, aunque es del $89,6 \%$ cuando las fibras se someten a agitación mecánica (Lindino, Marciniak, Gonzales Jr., \& Strey, 2014).
Otras investigaciones se han dedicado a observar la capacidad y los procesos de adsorción de la Luffa cylindrica al remover compuestos orgánicos complejos. En 2007 se reportó el uso de carbón activado de FLc en la remoción eficiente de reactivo naranja (RO) de una solución acuosa (Abdelwahab, 2007); en la Adenkule Ajasin University de Nigeria se reporta una eficiencia del $91 \%$ en la remoción de azul de metileno de aguas contaminadas y utilizando las FLc (Oladoja, Aboluwoye, \& Akunkugbe, 2008) y en 2009 la Universidad de Alejandría publicó a través del Journal of Enviromental Management, los resultados de su investigación en la remoción de colorante azul 106 de una solución acuosa utilizando carbón activado de Luffa egyptaca (Sayed \& Ashtoukhy, 2009).

En 2010, la Dokuz Eylül University de Turquía, reporta que al experimentar exitosamente las propiedades de la Luffa cylindrica en la remoción del verde de malaquita de una solución ligeramente ácida de agua (Altinisk, Gür, \& Seki, 2010), se realizó un tratamiento previo de las FLCs con $\mathrm{NaOH}$ al $1 \%$, se evaluaron las variables de temperatura, $\mathrm{pH}$ y tiempo de contacto y se estudió la cinemática del proceso; corroborando que uso del hidróxido de sodio en la preparación de las fibras provee de adecuados resultados para los fines adsorbentes del proceso tal como lo habían reportado Tanobe, Sidenstriker, Munaro \& Amico en 2004. También se determinó que la cinemática de la reacción es de segundo orden, estableciendo que el modelo que mejor se ajusta es el de Langmuir y Freundlich. Aspectos que habrían sido analizados por varias de las investigaciones de remoción con Luffa antes mencionadas y que se seguirán reportando por otros investigadores posteriores también citados en esta revisión.

Continuando con los tratamientos de remoción de colorantes, en Argelia se publicaron los resultados de un experimento de remoción de azul de metileno de aguas residuales, utilizando carbón activado elaborados a partir de FLc, en el que se reportaron eficiencias del $99 \%$ y del $82 \%$ según diferentes temperaturas (Cherifi, Fatiha, \& Salah, 2013), mientras que por su parte, desde el National Institute of Technology Calicut en la India, se informó 
que se efectuó la retención de colorantes disueltos en el agua residual de una industria textil, utilizando despojos fibrosos de la Luffa (Rangbhashiyam, Anu, \& Selvaraju, 2013).

Luego, en 2012, la Universidad Medea realiza una segunda experiencia en la que se estudió el proceso de adsorción estática de un sistema fenol/FLc en aguas residuales industriales (Henini, Laidani, \& Hanini, 2012); antes, en el laboratorio de biomateriales y fenómenos de transporte de la Universidad Dr. Yahia Farès de Médéa en Argelia se habrían efectuado pruebas de remoción de fenol de aguas residuales, utilizando cordones de Luffa como un novedoso adsorbente, reportando que al inspeccionar microscópicamente las fibras vegetales, éstas se apreciaban con los poros cubiertos por fenol (Cherifi, Hanini, \& Bentahar, 2008). En China, en la Henan Normal University, se realiza un experimento para remover 1-cetilpiridinio bromuro (CPB), tensoactivo empleado en la industria de la higiene oral y de fabricación de pesticidas, utilizando Luffa cylindrica; reportando resultados de eficiencia entre el $94,4 \%$ al $92,2 \%$ dependiendo de la superficie de contacto y la concentración del material adsorbente (Ye, Hu, \& Wang, 2013); en el mismo año también en Argelia se investiga en la Hassiba Ben Bouali University en la dinámica de adsorción del Fenol en un sistema compuesto por FLc con el fin lograr la comprensión de los procesos que intervienen en la adsorción dinámica del fenol sintético removido de aguas residuales industriales, en las FLc (Henini, Laidani, \& Hanini, 2012).

En Febrero de 2014 siguen interesando las investigaciones de remoción de fenol con FLc, en esta oportunidad el National Institute of Oceanography and Fisheries de la ciudad de Alejandría en Egipto reporta resultados dedicados a estudiar la cinética, la termodinámica y las isotermas de la reacción de difusión de microporos, concluyendo que la cinemática de la reacción depende de la temperatura, el tiempo de retención y el pH; y, que el modelo que mejor se ajusta a ella es el de Langmuir (Abdelwahab \& Amin, 2014), concordando con muchos investigadores anteriores que reportan similares resultados. Ampliando el campo de los estudios en adsorción de las FLc, en el laboratorio de química orgánica y bioquímica de la Université de Haute Alsace, en Francia, se demostró que las FLc son capaces de adherir nicotina del humo de tabaco (BouSaab, Boulanger, Schelenbaum, \& Neulist, 2013); en la Universidad de Alejandría se experimentó en la retención y limpieza de aceites minerales contaminantes del agua de mar, reportando que además de haber obtenido una alta eficiencia de remoción, se observaron una excelente selectividad de la fibra para diversos aceites minerales y una excelente repelencia del agua (Abdelwahab, 2013).

\section{Aplicación de las FLc en la inmovilización de microorganismos como sistema interactivo de tratamiento.}

Una de las aplicaciones mayormente exploradas por los científicos, corresponde a la realización de procesos que involucraron la inmovilización de microorganismos en la matriz esponjosa y fibrilar. Aparte de la importante propiedad adsorbente, característica de las fibras para retener iones metálicos pesados y de separar algunos compuestos orgánicos, las fibras de Luffa cylindrica son útiles para la creación de lechos para alojar ecosistemas microbianos para fines específicos: En un experimento en la Universidad de Unan en China, que involucró la inmovilización del hongo de la pudrición blanca, el Phanerochaete chrysosporium, se logró una adsorción del 90\% del Plomo (II) contaminante de una fuente de agua usada de muestra, con su consecuente desorción posterior (Xu et al., 2012). Este mismo hongo se utilizó en la remoción de colorantes utilizando como referente al Remazon azul brillante, tinte reactivo muy utilizado en la industria textil (Iqbal \& Saed, 2007), de igual manera en otra publicación, el investigador reportó que el mismo sistema es muy eficiente en la remoción de iones de Plomo (II), Cobre (II) y Zinc (II) (Iqbal \& Edyvean, 2003). Un grupo de investigadores taiwaneses y chinos, mediante la inmovilización del micro-alga Scenedesmus dimorphus, efectuaron experimentos de remoción de cadmio en agua, siendo otro aspecto muy interesante de esta investigación, el estudio que hace sobre las mejores condiciones de crecimiento celular en el soporte de esponja, para lograr un óptimo de remoción del metal 
(Chen et al.,2014). Inmovilizada en las FLc, se ha utilizado el alga verde unicelular Chlorella sorokiniana, en un tratamiento para la remoción de cadmio (Akhar, Saeed, \& lqbal, 2002); posteriormente, se utilizó el mismo sistema, en la remoción de Cromo (III) de aguas residuales industriales. Las investigadoras pakistaníes de esta investigación determinaron que el alga no solamente es eficiente en la remoción de $\mathrm{Cr}$ (III), sino que también establecieron que cuando está inmovilizada en FLc, la retención pasa de $4,97 \mathrm{mg} / \mathrm{g}$ a un máximo de $69,26 \mathrm{mg} / \mathrm{g}$ (Akhtar N. , Iqbal, Zafar, \& Iqbal, 2007).

Vale destacar que aparte de las referencias citadas, se han revisado otros reportes que describen experiencias de remoción de varios de los metales antes mencionados con la participación de micro-algas y hongos, llamando especialmente la atención de los investigadores, los tratamientos para remover cadmio. En otro ámbito, un grupo de investigadores mejicanos, experimentó la remoción de los plaguicidas organofosforados paratión metílico y coumafos, utilizando un consorcio bacteriano aislado de una muestra del suelo agrícola tomada en el estado de Morelos, en la región central de México y que fue cultivado en Luffa cylindrica. La experiencia reporta que se logró una eficiencia de remoción del 98\% y del $100 \%$, causado por el efecto combinado entre la actividad de los microorganismos, la adhesión a las células bacterianas y la adsorción al material de soporte, pues en pruebas hechas al consorcio bacteriano y a las FLc por separado, los resultados fueron similares en aproximadamente el $54.88 \%$ y $62 \%$ para el Paratión y el caumafos respectivamente (Moreno-Medina, Sánchez-Salinas, \& Ortiz-Hernandez, 2013), utilizando Chrysosporium phanerochaete inmovilizado en Luffa, sistema ya estudiado en la remoción de metales. También se reporta la remoción de aceite de oliva de aguas residuales (Ahmadi, Vahabzadeh, Bonakdarpour, Mehranian, \& Mofarrah, 2005).

La característica fibrosa y nano-tubular de las FLc se comportan de manera similar a las propiedades capilares y adsorbentes de algunos materiales sintéticos desarrollados para emular algunas de propiedades selectivas de los materiales naturales; así lo reportan Liu, Seki, Tanaka, \& Furusaky (1998) al comparar las eficiencias de remoción de las FLc con otras fibras sintéticas de poliuretano, prueba en la que se demostró una alta efectividad por parte de las fibras de diferentes secciones de la luffa para inmovilizar las sustancias de prueba, respecto de las capacidades de las fibras de poliuretano.

En el Instituto de Biodiseño de la Universidad Estatal de Arizona en Estados Unidos, se diseñó una membrana sintética, que dio origen a una tecnología de tratamiento de agua denominada MBfR (Membrane Biofilm Reactor). Esta membrana adsorbente, es capaz de retener sustancias minerales en sus microporos para facilitar los procesos de protonación liberadores de $\mathrm{H}+$; siendo que la especialidad particular de la MBfR es la de alojar a una comunidad sana de microorganismos dedicados a la degradación de sustancias orgánicas, haciendo muy completo el tratamiento para la potabilización de agua en el que han sido aplicadas (Arizona State University, 2013). Estas membranas MBfR son de muy reciente desarrollo sintético, sin embargo la propiedad inmovilizadora de microorganismos, además de las propiedades de adsorción y de protonación, entre otras adicionales, también las posee la esponja natural; que sin duda es una MBfR natural y ancestral. Evidencia de lo anterior se puede observar en investigaciones realizadas en bio-procesos que relacionan a las FLc con microorganismos inmovilizados y procesos de obtención o de separación de sustancias, como lo han demostrado los procedimientos descritos para inmovilización de cadmio II por bio-absorción continua con algas, la bio-adsorción de cromo (III) en aguas residuales industriales. Explica Ignacio Moreno-Garrido del instituto del Instituto de Ciencias Marinas de Andalucía (2007), que las fibras de luffa de diferentes especies han sido probadas en combinación con microorganismos tales como el hongo Phanaerochaete chrysosporium (Moreno, 2007), también se han usado la bacteria Zymomonas mobilis para la producción de sorbitol (Vignoli, Celligio, \& Silva, 2006) y la misma inmovilizada en celdas de Luffa para la producción de etanol (Behera, Mohanty, \& Ray, 2007). En 2006 el investigador Ruma Ganguly del Departamento de Botánica de la Universidad de Utkal en India reporta que mediante el uso del hongo Rhizopus 
oryzae inmovilizado en FLc se produjo ácido láctico (Ganguly, Dwivedi, \& Singh, 2006), incluso se han utilizado sistemas microbianos para la degradación de carbendazim y Ácido 2,4-diclorofenoxiacético (Nagase, y otros, 2007), entre otros muchos casos que podrían citarse. Finaliza Moreno Concluyendo que ya sea para fines civiles, comerciales o industriales las técnicas MBfR de inmovilización pasiva de colonias de algas en esponjas de lufa indica "ser un campo muy prometedor" (Moreno, 2007).

\section{Conclusiones}

Los tratamientos previos para la preparación de las FLc involucraron $\mathrm{NaOH}$ el cual mejora sus propiedades adsorbentes. La estructura fibrosa, esponjosa y nano-tubular de las fibras proveen de alta capacidad absorbente a las FLc. Las fibras son muy eficientes en la remoción de iones metálicos y de sustancias orgánicas complejas, mejorando la eficiencia del proceso, cuando se inmovilizan en ellas comunidades microbianas afines con el tratamiento.

Las FLc tienen alta capacidad de alojar comunidades de algas, hongos o bacterias. Las FLc tienen una moderada capacidad para retener sustancias minerales no metálicas en los tratamientos de desalinización. Sin embargo, esta condición mejora cuando los tratamientos se hacen por percolado longitudinal a través ductos contenedores de fibras trozadas entre 1 y $2 \mathrm{~mm}$ de longitud.

Los investigadores refieren una alta eficiencia en la desorción de las fibras, de minerales y de metales, en un caso; o en la mineralización de compuestos orgánicos en otro; como tratamiento posterior a la remoción. En los tratamientos reportados, la obtención de las isotermas para el análisis de las eficiencias de remoción, la cinemática del proceso y su termodinámica, se reportaron con mejores resultados aquellas que utilizaron los modelos de Langmuir \& Freundlich y que consideraron reacciones de primero y segundo orden. La alta tasa de adsorción indica la viabilidad de utilizar esta biomasa en la remediación de ambientes contaminados.
Esta investigación evidencia la posibilidad de utilizar fibras de Luffa cylindrica para el tratamiento de agua potable y de aguas residuales industriales, destacando que sus ventajas adicionales se refieren a su economía, no toxicidad, sostenibilidad y biodegradabilidad. La Luffa cylindrica es un bio-adsorbente eficaz, capaz de retener metales pesados en sus nano-fibras. En este sentido, el estropajo ofrece una opción barata y tecnológicamente accesible para el tratamiento de agua potable y de aguas residuales.

\section{Agradecimientos}

Los autores expresan su agradecimiento al grupo de investigación ESLINGA del programa de Ingeniería Industrial de la Universidad Cooperativa de Colombia y al programa de Maestría en Ingeniería Ambiental de la Universidad Mariana.

\section{Literatura citada}

1. Adie, D., Igboro, S., \& Daouda, N. (2013). Determination of the Filter Potential of Luffa Sponge (luffa aegyptiaca) in Water Quality Analysis. American International Journal of Contemporary Research. Recuperado de http://www.aijcrnet.com/journals/Vol_3_ No_3_March_2013/11.pdf

2. Ahmadi, M. et al. (2005). Phenolic removal in olive oil mill wastewater using loofah immobilized loofahimmobilized. World Journal of Microbiology and Biotechnology.: Recuperado de: http://link.springer.com/article/1 0.1007\%2Fs11274-005-9006-3

3. Akhtar, N., Iqbal, J., \& Iqbal, M. (2004). Removal and recovery of nickel(II) from aqueous solution by loofa sponge-immobilized biomass of Chlorella sorokiniana: characterization studies. Journal of Hazardous Materials. Recuperado de: www.elseiver.com/locate/ hazmat

4. Altinisk, A., Gür, E. \& Seki, Y. (2010). Luffa cylindrica for removal of a model basic dye. Journal of Hazardous Materials. Anatural sorbent. Recuperado de: http://www. journals.elsevier.com/journal-of-hazardous-materials

5. Aluyor, E., Oboh, I. \& Adhu, T. (2009). Post-treatment of Produced water before discharge using luffa cylindrica. Electronic Journal of Practices and Technologies. Recuperado de: http://lejpt.academicdirect.org/ A14/057_064.pdf

6. ASU, Arizona State University. (2013). Cleaning water with nature. ASU Biodesing Institute. Recuperado de: http://www.biodesign.asu.edu/research/projects/cleaning-water-with-nature 
7. Azeez, M. A., Belllo, O. S. \& Adedeji, A. O. (2013). Traditional and medicinal uses of Luffa cylindrica: a review. Journal of Medicinal Plants Studies. Recuperado de.: http://www.plantsjournal.com/vol1lssue1/lssue_ sep_2013/12.1..pdf

8. Behera, S., Mohanty, R. \& Ray, R. (2007). Ethanol fermentation of sugarcane molasses by Zymomonas mobilis mtcc 92 immobilized in Luffa cylindrica I. sponge discs and ca-alginate matrices. Recuperado de NCBI: http://www.ncbi.nlm.nih.gov/pubmed/24031981

9. Bou-Saab, H., Boulanger, A., Schelenbaum, P. \& Neulist, S. (2013). Performance of Luffa cylindrica as immobilization matrix in bioconversion reactions by Nicotina tabacum BY-2. Journal of Bioscience and Bioengieering. Recuperado de: www.elsevier.com/locate/jbiosc

10. Boynard, C., Monteiro, S. \& d'Almeida, J. (2002). Aspects of alkali tratment of sponge ground (Luffa cylindrica) fibers on the flexural propieties of polyester matrix composites. Wiley Online Library. Recuperado de: http://onlinelibrary.wiley.com/doi/10.1002/app.11522/ abstract;jsessionid=287D991757085601B024A569D6 2C0C78.f04t01?deniedAccessCustomisedMessage $=$ \&userlsAuthenticated=false

11. Chen, B.-Y. et al., (2014). Fixed-bed biosorption of cadmium using immobilized Scenedesmus obliquus CNW-N cells on loofa (Luffa cylindrica) sponge. Science Direct Bioresource technology. Recuperado de.: www.elsevier.com/locate/biortech

12. Cifuentes, B. \& Pataquiva, L. (2013). Uniuversidad de la Sabana. Pretratamiento fotocatalítico para la obtención de alcohol celulósico a partir de Luffa cylindrica:: Recuperado, de http://intellectum.unisabana.edu. co:8080/jspui/bitstream/10818/10755/1/LAURA\%20 PATAQUIVA\%20(T)\%20TESIS.pdf

13. Ganguly, R., Dwivedi, P. \& Singh, R. (2006). Production of lactic acid with loofa sponge immobilized Rhizopus oryzae RBU2-10. Science Direct Recuperado de: http://www.sciencedirect.com/science/article/pii/ S0960852406001982

14. Iqbal, M. \& Edyvean, R. (2003). Biosorption of lead, copper and zinc ions on loofa sponge immobilized biomass of Phanerochaete chrysosporium. Science Direct. Recuperado de: http://www.sciencedirect.com/ science/article/pii/S0892687503004102

15. Iqbal, M. \& Saeed, A. (2007). Biosorption of reactive dye by loofa sponge-immobilized fungal biomass of Phanerochaete chrysosporium. Science Direct. Recuperado de: http://www.sciencedirect.com/science/ article/pii/S1359511307001444

16. Kaewta, K. \& Thongmee, J. (2012). Studies on the structura and propietes of thermoplastic strach/luffa fiber composites. Materials and Design. Recuperado de: http://aplicacionesbiblioteca.udea.edu.co:2063/ science/article/pii/S0261306912002270

17. Laidan, Y., Hanini, S., \& Henini, G. (2010). Valorization of Luffa cylindrica for water treatments copper chargers. Study of the possibility of regeneration by chermical desorption. Oman: Sultan Qaboos University.

18. Lindino, C. A., Marciniak, A. A., Goncalvez Jr, A. \&
Strey, L. (2014). Adsorption of cadmium in vegetable sponge (Luffa cylindrica). Ambiente \& Água - An Interdisciplinary Journal of Applied Science.

19. Liu, Y.-K., Seki, M., Tanaka, H. \& Furusaky, H. (1998). Characterristics of Loofa (Luffa cylindrica) sponge as a carrier for plant cell immobilization. Journal of fermentation and bioengineering, 416.

20. López, A., Vasquez, Suárez, A. \& Gómez, C. (2012). Assessment of dye adsorption by Luffa Cylindrica fibers using experimental design methodology IA ENG International Association of Engineers. Recuperado de: http://www.iaeng.org/publication/WCE2012/ WCE2012_pp646-650.pdf

21. Moreno, I. (2007). Effect of environmental factors on performance performance of immobilized consortium system for degradation of carbedazim and 2,4-dichlorophenoxyacetic acid in continuous culture. España: Science Direct Bioresourse Technology.

22. Moreno, I. (España, 2007). Microalgae imobilization: current techniques and uses. Science Direct Bioresourse Technology.

23. Moreno-Medina, D., Sánchez-Salinas, E., \& OrtizHernandez, M. (Octubre de Méjico, 2013). Removal of methyl parathion and coumaphos pesticides by a bacterial consortium immobilized in Luffa cylindrica. E-Journals UNAM. Recuperado de: http://www.journals.unam. mx/index.php/rica/article/download/35799/40214.

24. Nagase, H. et al. (2006). Effect of enviromental factors on performance of inmobilized consortium system for degradation of carbedazim and 2,4-dichlorophenoxyacetic acid in continuos culture. Biochemistry Process.

25. Nasreen, A. et al. (2007). Biosorption characteristics of unicellular green alga Chlorella sorokiniana immobilized in loofa sponge for removal of $\mathrm{Cr}(\mathrm{III})$. NCBI. Recuperado de: http://www.ncbi.nlm.nih.gov/pub$\mathrm{med} / 18574966$

26. Piao Xu et al. (2012). Adsorption of $\mathrm{Pb}$ (II) by iron oxide nanoparticles immobilized Phanerochaete chrysosporium: Equilibrium, kinetic, thermodynamic and mechanisms analysis. Chemical Engineering Journal. Recuperado: http://ee.hnu.cn/eeold/php/news/pic/yunfeirandompic_1344651287.pdf

27. Purkayastha, D., Mishra, U. \& Biswas, S. (2014). A comprehensive review on $\mathrm{Cd}$ (II) removal from aqueous solution. Journal of Water Process Engineering Recuperado de: www .els evier .c om /lo cat e/jw p e

28. Shajidi, A., Jalilnejad, N. \& Jalilnejad, E. (2013). A study on adsorption of cadmium(II) ions from aqueous solution using Luffa cylindrica. The online platform for Taylor \& Francis Group content. Recuperado de: http:// www.tandfonline.com/doi/abs/10.1080/19443994.2013 .873878\#.VG1Zm9KG9jY

29. Shapiro, L. (2012). EOL Encyclopedia of Life. Recuperado de Luffa aegyptiaca: Recuperado de: http://eol. org/data_objects/22472617

30. Shen, J. \& et al. (2012). Mechanical properties of luffa sponge. SciVerse Science Direct.: Recuperado de: http://aplicacionesbiblioteca.udea.edu.co:2063/science/article/pii/S1751616112001993 
31. Shen, J., Xie, Y. M., Huang, X., Zohu, S. \& Ruan, D. (2013). Behaviour of luffa sponge material under dynamic loading. International Journal of Impact Engineering: Recuperado de http://www.sciencedirect.com/ science/article/pii/S0734743X13000110

32. UNAD. (2013). Lección 29. Aspectos botánicos y labores cultivo del estropajo. Recuperado de: http:// datateca.unad.edu.co/contenidos/303022/Modulo_ del_curso_en_exe/leccin_29_aspectos_botnicos_y_ labores_cultivo_del_estropajo.html
33. Vignoli, J. A., Celligio, M. \& Silva, R. (2006). Development of a statistical model for sorbitol production by free and immobilized Zymomonas nobilis in a luffa saponge Luffa Cylindrica. Processn Biochem.

34. Ye, C., Hu, N. \& Wang, Z. (2013). Experimental investigation of Luffa Cylindrica as a natural sorbent material for the removal of cationic surfactant. ScienceDirect Journal of the Taiwan of Chermical Engineers. Recuperado de: http://www.sciencedirect.com/science/article/ pii/S1876107012001344
Conflicto de Intereses Los autores declaran no tener ningún conflicto de intereses

Recibido: septiembre 09 de 2016 Aceptado: octubre 14 de 2016 
